\title{
Descripción de un proceso participativo de diagnóstico de la institucionalización del aprendizaje-servicio
}

\author{
I. Salterain, A. Eizaguirre, A. Díaz-Iso, M. Aláez Martínez, M. García-
} Feijoo, M. Roldán-Henao. Universidad de Deusto, Bilbao

L. Mota Ribeiro. Universidade Católica de Portugal, Portugal

P. Booth. Universidad de St. Mary 's, Londres, Inglaterra

D. Simeone. Università Cattolica del Sacro Cuore, Roma, Italia

\section{Resumen}

Para avanzar en la institucionalización del aprendizaje-servicio (ApS) en las universidades católicas, en 2020 se llevó a cabo el lanzamiento del programa UNISERVITATE. Este artículo tiene como objetivo describir cómo se aborda un proceso participativo de diagnóstico de la institucionalización del ApS a partir de la rúbrica de Furco en St. Mary's university, Universidade Católica de Portugal y Università Cattolica del Sacro Cuore. Dicho proceso ha sido liderado por el hub de la región, la Universidad de Deusto. Para ello se ha llevado a cabo una evaluación del grado de institucionalización del ApS a través de una herramienta que identifica en qué punto del proceso de institucionalización se encuentran las universidades. Como resultado se presenta la herramienta, las fortalezas y debilidades identificadas en las instituciones, y los planes de acción derivados de estas. Finalmente, se pone de relieve la necesidad de abordar investigaciones sobre lo que supone la institucionalización del ApS en las instituciones de educación superior católicas, así como la importancia de potenciar dicho proceso para promover el desarrollo humano integral del alumnado en educación superior.

\section{Palabras clave}

Aprendizaje-servicio, rúbrica, institucionalización, nodo sur de Europa, Uniservitate.

Fecha de recepción: 24/XI/2021

Fecha de aceptación: 9/XII/2021 


\title{
Description of a participatory diagnostic process of the institutionalization of service-learning
}

\begin{abstract}
To advance the institutionalization of service-learning (SL) in Catholic universities, the launch of the UNISERVITATE program was carried out in 2020. The objective of this article is to describe how a participatory process for diagnosing the institutionalization of SL is approached from the Furco rubric at St. Mary's university, Universidade Católica de Portugal and Università Cattolica del Sacro Cuore. This process has been led by the region's hub, the University of Deusto. To this end, an evaluation of the degree of institutionalization of the SL has been carried out through a tool that identifies where the universities are in the institutionalization process. As a result, the tool, the strengths and weaknesses identified in the institutions, and the action plans derived from them are presented. Finally, the need to undertake research on what the institutionalization of SL implies in Catholic higher education institutions is highlighted, as well as the importance of promoting said process to promote the integral human development of students in higher education.
\end{abstract}

\section{Key words}

Service-learning, rubric, institutionalization, southern node of Europe, Uniservitate. 


\section{Descripció d'un procés participatiu de diagnòstic de la institucionalització de l'aprenentatge servei}

\section{Resum}

Per avançar en la institucionalització de l'aprenentatge servei (ApS) a les universitats catòliques, el 2020 es va dur a terme el llançament del programa UNISERVITATE. Aquest article té com a objectiu descriure com s'aborda un procés participatiu de diagnòstic de la institucionalització de l'ApS a partir de la rúbrica de Furco a la St. Mary's University, la Universidade Catòlica de Portugal i la Università Cattolica del Sacro Cuore. Aquest procés ha estat liderat pel hub de la regió, la Universitat de Deusto. Per això s'ha dut a terme una avaluació del grau d'institucionalització de l'ApS a través d'una eina que identifica en quin punt del procés d'institucionalització es troben les universitats. Com a resultat es presenta l'eina, les fortaleses i les debilitats identificades a les institucions, i els plans d'acció que se'n deriven. Finalment, es posa en relleu la necessitat d'abordar investigacions sobre el que suposa la institucionalització de l'ApS a les institucions d'educació superior catòliques, així com la importància de potenciar aquest procés per promoure el desenvolupament humà integral de l'alumnat en educació superior.

\section{Paraules clau}

Aprenentatge servei, rúbrica, institucionalització, node sud d’Europa, Uniservitate. 


\section{Introducción}

Actualmente la sociedad se enfrenta a retos complejos de índole social, político, económico y medioambiental. Ante esta situación, es razonable pensar que los problemas deben abordarse desde diversos planteamientos. Por ello, las instituciones de educación superior tienen el cometido de formar personas que sepan encontrar soluciones nuevas a los presentes y futuros problemas (Aktas et al., 2015).

Las universidades están inmersas en nuestra sociedad y son agentes clave para solucionar los diferentes retos a los que nos enfrentamos. En esta línea, la Organización de las Naciones Unidas para la Educación, la Ciencia y la Cultura (UNESCO) en 2017, planteó los objetivos de desarrollo sostenible entre los que destaca la necesidad de hacer frente a la pobreza e impulsar la inclusión social. Las universidades católicas de todo el mundo están especialmente convocadas a participar en este reto. De hecho, la Compañía de Jesús ha expresado su preocupación también sobre la necesidad de dar respuesta a los desafíos sociales, y para ello, ha puesto en marcha las Preferencias Apostólicas Universales (Sosa, 2019) con el propósito de orientar el trabajo de la Compañía hasta el año 2029. Estas Preferencias tienen como fin ayudar a las instituciones desde una misión ignaciana (basadas en experiencias espirituales y sociales) y así poder colaborar en la formación y apoyo integral de los jóvenes.
Para lograr ser parte del cambio social y contribuir a los diferentes retos sociales, se requiere de ciertos cambios en los modelos de enseñanzaaprendizaje en las instituciones de educación superior. Estos modelos de enseñanza han de impactar socialmente y ayudar a formar una ciudadanía activa capaz de llevar a cabo la transformación social (Díaz-Iso et al., 2019).

En esta concepción de la educación superior se considera la propuesta pedagógica de aprendizaje-servicio como un ámbito privilegiado para contribuir no solo al desarrollo integral del alumnado, sino también para el reto de crear ciudadanía activa, justa y solidaria (Pena Mardaras, 2020; Tapia, 2010). A nivel internacional hay numerosas evidencias científicas que demuestran la potencialidad e influencia del aprendizaje-servicio tanto en el ámbito académico del alumnado, como en el desarrollo profesional (Hart, 2015; Salam et al., 2019; Song et al., 2018). Esto se debe a su idoneidad para promover habilidades sociales y reflexivas que son perfectamente adaptables a contextos laborales, sociales o académicos (Mason y Dunens, 2019).

El aprendizaje-servicio ha sido definido de distintas formas, incluso se ha llegado a confundir con otros servicios solidarios, como el voluntariado o prácticas profesionales. La literatura científica publicada hasta el momento nos revela que varios enfoques han influido en el modelo de aprendizaje que se lleva a cabo en las prácticas de aprendizaje-servicio (García-Romero y Lalueza, 2019). Entre ellas, el modelo 
de aprendizaje experiencial de John Dewey (1933) pone de relieve la importancia de realizar una reflexión sobre la acción; la pedagogía de Freire (2018) hace hincapié en el desarrollo de la conciencia crítica; y Mezirow (1991) en el aprendizaje transformativo. Furco (1996) marca la diferencia del aprendizaje-servicio de otros conceptos y argumenta que para que una práctica se pueda considerar aprendizaje-servicio es necesario que haya un equilibrio equitativo entre el servicio que se presta para dar respuesta a una necesidad social como en el aprendizaje del alumnado. Otros autores como Schön (1983) la distinguen por la interrelación que existe entre el pensar y hacer en las experiencias de aprendizaje-servicio. Es decir, hay una mayor comprensión y conocimiento a partir de experiencias diarias y al mismo tiempo nos invita a reflexionar ideas, a la búsqueda de nuevas ideas y resolver dificultades (Páez y Puig, 2013).

Por lo tanto, el aprendizaje-servicio es una pedagogía educativa innovadora en la que se combina aprendizaje en el aula al mismo tiempo que se dan nuevos conocimientos en contextos reales (Bringle y Hatcher, 1995; Tapia, 2010) a través de un servicio comunitario. Se trata de poder transferir el conocimiento en un entorno real para responder a los desafíos comunitarios actuales, crear vínculos sociales, mejorar la convivencia y el crecimiento personal de cada individuo (Hart, 2015; Salam et al.,2019). De esta manera, favorece en el alumnado un desarrollo activo, responsable, comprometido, reflexivo y con una serie de valores efectivos para hacer frente a diferentes retos sociales (Mason y Dunens, 2019; Asghar y Rowe, 2016).

Hay distintas maneras de incorporar el aprendizaje-servicio en el plan de estudios de educación superior, es decir, se puede llevar a cabo a través de proyectos curriculares, actividades extracurriculares o proyectos de investigación (Bringle y Hatcher, 1995). Sin embargo, la institucionalización del aprendizaje-servicio en las instituciones de educación superior está presentando grandes dificultades, entre ellas se destaca la falta de proyectos a largo plazo, la insuficiente formación del profesorado y personas en puestos de dirección dentro de la institución, la falta de fondos económicos para poner y sostener las experiencias de aprendizaje-servicio, etc. (SantosPastor et al.,2020; Capella-Peris et al., 2019; Salam et al., 2019). Por ello, para muchas universidades la institucionalización del aprendizajeservicio es todavía un reto.

Esta comunicación tiene como objetivo describir cómo se puede abordar un proceso participativo de diagnóstico de la institucionalización del aprendizajeservicio en una institución concreta a partir de la Rúbrica de Furco (2003). Más concretamente, se explica el proceso llevado a cabo con St. Mary's university (en Reino Unido), Universidade Católica de Portugal (en Portugal) y Università Cattolica del Sacro Cuore (en Italia).

Para ello, en este artículo primero se explica la importancia de la institucionalización del aprendizajeservicio en las instituciones de educación superior. Después, se lleva a

Salterain, I., Eizaguirre, A., Díaz-Iso, A., Aláez Martínez, M., García-Feijoo, M., Roldán-Henao, M., Mota Ribeiro, L., Booth, P. y Simeone, D. (2021). Descripción de un proceso participativo de diagnóstico de la institucionalización del aprendizaje-servicio. RIDAS, Revista Iberoamericana de Aprendizaje Servicio, 12, 149-162. DOI10.1344/RIDAS2021.12.15 
cabo una descripción de la herramienta de autoevaluación institucional de aprendizaje-servicio basada en la Rúbrica de Furco (2003) y creada por la Universidad de Deusto. A continuación, se detallan las 5 dimensiones con sus indicadores pertenecientes.

Posteriormente, se relata paso por paso la metodología que hemos llevado a cabo para el proceso de diagnóstico de la institucionalización del aprendizajeservicio en las tres universidades. Tras la metodología y utilización de dicha herramienta, se comentan las fortalezas y las debilidades detectadas en cada universidad. Para finalizar, se presenta la imagen global de autoevaluación y se narra el Plan de Acción propuesta para cada universidad.

\subsection{Importancia de institucionalizar el} aprendizaje-servicio en las instituciones de educación superior

Para lograr que la metodología de aprendizaje-servicio sea eficiente y aporte valor en las instituciones de educación superior, en particular, y en la sociedad, en general, esta debe estar institucionalizada. Institucionalizar significa un proceso en el que la universidad aplica esta práctica de forma estructurada y sustentada por todos los agentes implicados (Lewing y Shehane, 2017). Se ha de integrar en el currículo del estudiante, implicar a los socios comunitarios en los proyectos de aprendizaje-servicio, llevar a cabo formaciones para profesorado y equipos directivos de cómo implementar la metodología, dar apoyo institucional reconociendo y promocionando al docente que participa en experiencias de aprendizaje-servicio, invertir fondos económicos para garantizar la sostenibilidad de las experiencias, etc. (Lewing y Shehane, 2017).

Este proceso de institucionalización ha de llevarse a cabo de manera gradual. En esta línea, Furco (2003) señala tres etapas en el proceso de institucionalización: Creación de Masa Crítica, Construcción de Calidad e Institucionalización Sostenible. La primera etapa, Creación de Masa Crítica, se da cuando las instituciones de educación superior empiezan a tomar en consideración el aprendizajeservicio y a crear apoyo institucional. La segunda etapa, Construcción de Calidad, se da cuando las instituciones se centran en el desarrollo de actividades de aprendizaje-servicio de calidad y se presta mayor atención a la calidad de las actividades que a la cantidad de actividades. Por último, la tercera etapa, Institucionalización Sostenible, se da cuando la universidad tiene totalmente integrada la institucionalización del aprendizajeservicio.

\subsection{Herramienta de autoevaluación} institucional de aprendizaje-servicio basado en la Rúbrica de Furco (2003)

La Rúbrica de autoevaluación de Furco (2003) es una guía creada con el fin de medir el nivel en el que se encuentra cada universidad en el proceso de institucionalización del aprendizajeservicio. Es una rúbrica que permite reconocer las fortalezas y debilidades de cada institución y en qué fase o etapa se encuentra. Por ello, se considera una herramienta imprescindible para poder 
institucionalizar el aprendizaje-servicio en las universidades.

Tal y como hemos mencionado anteriormente, la Rúbrica de Furco (2003) distingue tres etapas fundamentales:

- Etapa I Creación de Masa Crítica;

- Etapa II Construcción de Calidad;

- Etapa III Institucionalización Sostenible.

Al mismo tiempo divide el proceso de institucionalización en cinco dimensiones diferentes:

1. Filosofía y Misión del Aprendizaje-Servicio;

2. Involucramiento y Apoyo de los Docentes en el AprendizajeServicio;

3. Involucramiento y Apoyo de los Estudiantes en el AprendizajeServicio;

4. Participación y Asociación de los Socios Comunitarios;

5. Apoyo Institucional al Aprendizaje-Servicio (Figura 1).
Figura 1. Las 5 dimensiones clave de la institucionalización del aprendizaje-servicio

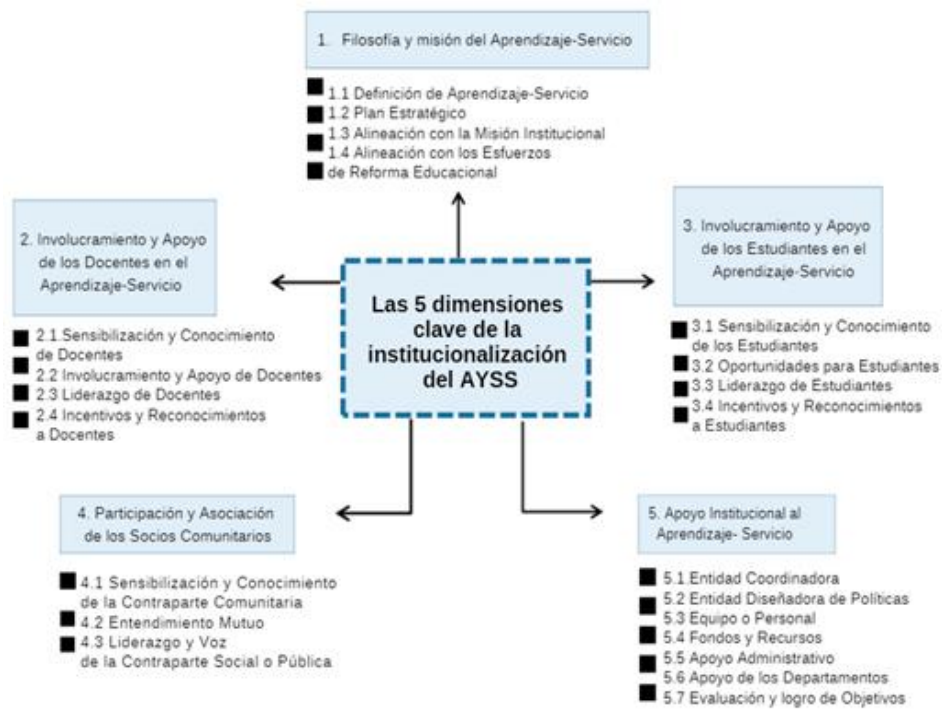

Fuente: elaboración propia a partir de Furco (2003)

A través de este instrumento se trata de que cada universidad reflexione sobre el apoyo que posee en estas dimensiones para fomentar el aprendizaje-servicio. Esta autoevaluación permite a las distintas instituciones a desarrollar un Plan de Acción sobre el aprendizaje-servicio, en base a las necesidades detectadas anteriormente (Furco, 2003).

\subsubsection{Explicación detallada de las 5 dimensiones y los indicadores que las componen}

Respecto a la primera dimensión (Filosofía y Misión del Aprendizaje-Servicio), en un primer indicador tienen que evaluar en qué etapa se encuentran a la hora de definir el aprendizaje-servicio en sus instituciones. En un segundo indicador, se les pregunta si la planificación 
estratégica de la universidad integra el aprendizaje-servicio y cuáles son las metas u objetivos principales para ello. En un tercer indicador, tienen que reflexionar de qué manera está el aprendizaje-servicio incluido en la filosofía y misión de la universidad. En un cuarto indicador, tienen que evaluar cómo se integra el aprendizaje-servicio a la hora de reflexionar sobre cómo abordar los retos educativos.

En cuanto a la segunda dimensión (Involucramiento y Apoyo de los Docentes en el Aprendizaje-Servicio) tienen que centrarse en el papel que juegan los docentes en el aprendizajeservicio en su centro. Por un lado, en un primer indicador, hablan acerca de la sensibilización que expresan los docentes hacia el aprendizaje-servicio. En un segundo indicador, tratan acerca de la implicación que estos tienen. En un tercer indicador, se les pregunta si los docentes implicados en los proyectos de aprendizaje-servicio son personas influyentes en la universidad, y por último, en un cuarto indicador, se les cuestiona si los docentes involucrados en el aprendizaje-servicio reciben algún incentivo 0 reconocimiento.

En relación a la tercera dimensión (Involucramiento y Apoyo de los Estudiantes en el Aprendizaje-Servicio) era similar a la anterior, pero, esta vez, evalúan el rol de los estudiantes en la institucionalización del aprendizajeservicio. Por lo tanto, en un primer indicador, se les pregunta cómo se da a conocer en sus centros los proyectos de aprendizaje-servicio a los estudiantes. En un segundo indicador, se les cuestiona si las experiencias que se ofrecen de aprendizaje-servicio son variadas y numerosas para los diferentes perfiles de estudiantes que hay en la universidad. En un tercer indicador, tienen que evaluar el papel que asumen los estudiantes en las prácticas de aprendizaje-servicio. En un cuarto indicador, reflexionan sobre qué incentivos o reconocimientos reciben los estudiantes involucrados en el aprendizaje-servicio.

Ahora bien, la cuarta dimensión (Participación y Asociación de los Socios Comunitarios) trata sobre la participación y asociación con socios comunitarios. En un primer indicador, tienen que evaluar cómo se articula la relación de la universidad con los socios de la comunidad. Y de manera similar a los casos anteriores, debe haber sensibilización y conocimiento de las características e implicaciones de la metodología. En un segundo indicador, se les pregunta si se acuerda con los socios comunitarios los objetivos de los proyectos de aprendizaje-servicio. En un tercer indicador, se les cuestiona de qué manera los socios comunitarios contribuyen activamente a la institucionalización del aprendizajeservicio.

Por último, en la quinta dimensión (Apoyo Institucional al Aprendizaje-Servicio) se recogen los aspectos operativos relacionados con la gestión, organización y administración del proceso. Esta dimensión incluye, en un primer indicador, si hay un equipo que coordina y gestiona la institucionalización del aprendizajeservicio. En un segundo indicador, se les cuestiona de qué manera reconoce la dirección del centro el aprendizaje- 
servicio como un objetivo esencial para la educación superior. En un tercer indicador, tienen que evaluar si hay recursos personales dedicados al aprendizaje-servicio. En un cuarto indicador, se les pregunta sobre la existencia de recursos económicos dedicados al aprendizaje-servicio. En un quinto indicador, se les pregunta en cuántas titulaciones o facultades está integrado el aprendizaje-servicio como parte formal del programa. En un quinto indicador, tienen que integrar si hay alguna herramienta diseñada para evaluar de manera sistemática el desarrollo de las actividades de aprendizaje-servicio.

\section{Metodología}

Tal y como se ha comentado previamente, esta comunicación tiene como objetivo describir cómo se puede abordar un proceso participativo de diagnóstico de la institucionalización del aprendizaje-servicio en una institución concreta a partir de la Rúbrica de Furco (1999).

Más concretamente, abordaremos cómo se ha llevado a cabo el proceso de diagnóstico de la institucionalización del aprendizaje-servicio solidario en St. Mary's University (en Reino Unido), Universidad de Católica Portuguesa (en Portugal) y Università Cattolica del Sacro Cuore (en Italia).

Para abordar el objetivo, se ha llevado a cabo una evaluación del grado de institucionalización de aprendizajeservicio en las tres universidades mencionadas. Esta evaluación se ha llevado a cabo en tres pasos diferentes y se ha realizado a través de la herramienta para la autoevaluación anteriormente descrita.

Figura 2. Procedimiento llevado a cabo para el diagnóstico de la institucionalización

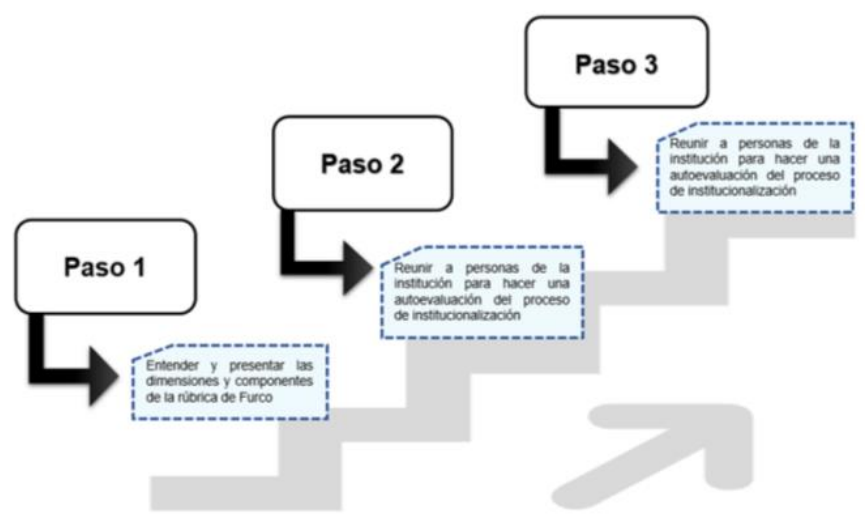

Fuente: elaboración propia

Como todo proceso, antes de dar cualquier paso, hicimos una planificación detallada y organizamos las tareas que íbamos a ejecutar en diferentes fechas. Primero, tal y como se puede observar en la Figura 2, comprendimos las dimensiones y componentes de la Rúbrica de Furco (Figura 2) y llevamos a cabo una reunión regional explicando la herramienta al equipo de trabajo de las tres universidades.

En segundo lugar, pedimos a cada institución que formarán un equipo de trabajo para hacer una autoevaluación del proceso de institucionalización. Para ello, por cada dimensión, diseñamos una tabla dividida en tres etapas diferentes (Figura 3) para que cada universidad pudiera reflexionar en qué etapa de institucionalización se 
encontraba (Etapa I: Creación de Masa Crítica; Etapa II: Construcción de Calidad; Etapa III: Institucionalización Sostenible y cada dimensión). En tercer lugar, reunimos a los equipos de trabajo de cada institución para que nos presentaran la autoevaluación que había llevado a cabo, así como las fortalezas y debilidades en cada universidad.

Figura 3. Ejemplo de la Dimensión 1 (Filosofía y Misión del Aprendizaje-Servicio) de la herramienta

\begin{tabular}{|l|l|l|l|}
\cline { 2 - 4 } \multicolumn{1}{c|}{} & Etapa I & Etapa II & Etapa II \\
\hline Definición de AYSS & & & \\
\hline Plan Estratégico & & & \\
\hline $\begin{array}{l}\text { Alineación con la } \\
\text { misión institucional }\end{array}$ & & & \\
\hline $\begin{array}{l}\text { Alineación con los } \\
\text { esfuerzos de reformas } \\
\text { educacionales }\end{array}$ & & & \\
\hline $\begin{array}{l}\text { Valoración global } \\
\text { dimensión 1 }\end{array}$ & & & \\
\hline
\end{tabular}

Fuente: elaboración propia a partir de Furco (2003)

\section{Resultados y discusión}

Tras utilizar la herramienta de autoevaluación del proceso de institucionalización, recopilamos información sobre las fortalezas y debilidades detectadas en cada universidad y agrupamos en qué etapa se encontraba cada una de ella por cada dimensión. En primer lugar, tal y como se puede observar en la Figura 4, cabe destacar que todas las universidades están en proceso de institucionalizar el aprendizaje-servicio, porque las tres universidades se encuentran, por cada dimensión, en la primera o segunda etapa.

Figura 4. Imagen global de la etapa en la que se encuentra cada universidad (Reino Unido, Italia y Portugal)

\begin{tabular}{|c|c|c|}
\hline Etapa 1 & Etapa 2 & Etapa 3 \\
\hline $\begin{array}{l}\text { 1. Filosofia y Misión del } \\
\text { 2. Aprendizaje-Servicio } \\
\text { Involucramiento y Apoyo } \\
\text { de los Docentes en el } \\
\text { AYSS } \\
\text { 3. Involucramiento y Apoyo } \\
\text { de los Estudiantes en el } \\
\text { AYSS } \\
\text { 4. Participación y } \\
\text { Asociación de los Socios } \\
\text { Comunitarios } \\
\text { (comunitaria) } \\
\text { 5. Apoyo Institucional al } \\
\text { Aprendizaje-Servicio }\end{array}$ & $\begin{array}{l}\text { 1. Filosofia y Misión del } \\
\text { Aprendizaje-Servicio } \\
\text { 2. Involucramiento y Apoyo } \\
\text { de los Docentes en el } \\
\text { AYSS } \\
\text { 3. Involucramiento y Apoyo } \\
\text { de los Estudiantes en el } \\
\text { AYSS } \\
\text { 4. Participación y Asociación } \\
\text { de los Socios } \\
\begin{array}{l}\text { Comunitarios } \\
\text { (comunitaria) }\end{array} \\
\text { 5. Apoyo Institucional al } \\
\text { Aprendizaje-Servicio }\end{array}$ & 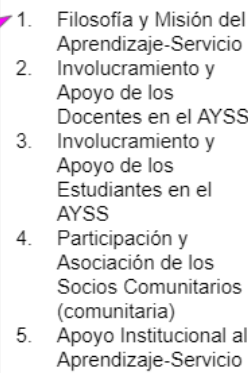 \\
\hline
\end{tabular}

Fuente: elaboración propia a partir de Furco (2003)

Respecto a los resultados obtenidos a través de la herramienta en la primera dimensión, a pesar de que en alguna institución existe una definición para el aprendizaje-servicio, la mayoría no tienen una definición institucionalizada. También queda al descubierto la necesidad de trabajar en un plan estratégico oficial para impulsar el aprendizaje-servicio.

En cuanto a la segunda dimensión, la que se refiere al papel que juega el docente en la institucionalización del aprendizaje-servicio, sigue siendo necesario aumentar esfuerzos para ayudar al profesorado a distinguir el aprendizaje-servicio de otras prácticas de aprendizaje experiencial. Hay cierta incomprensión sobre la metodología y conocimiento, por lo que se necesitan instructores y líderes preparados para poder impulsar el aprendizaje-servicio. 
Resulta revelador también que las tres universidades destacan la importancia de que haya un reconocimiento a nivel institucional al profesorado que se involucra en prácticas de aprendizajeservicio. Para avanzar en la institucionalización este apoyo es imprescindible puesto que el aprendizaje-servicio no ha de depender únicamente de la voluntad individual y el compromiso personal del profesorado.

A diferencia de lo que pasa con los docentes, parece que en las tres instituciones existe mayor conocimiento por parte del alumnado sobre la metodología aprendizaje-servicio, sus beneficios, el rol que han de jugar, etc. Las tres instituciones destacan que tienen mayores recursos y oportunidades disponibles para ellos y están informados sobre las distintas iniciativas de aprendizaje-servicio que se llevan a cabo en la universidad. No obstante, en el proceso de autoevaluación las instituciones destacan la importancia de poner más oportunidades a su disposición para que participen en experiencias y en diferentes proyectos de aprendizajeservicio. Para ello, es crucial que esté integrado en el plan curricular del estudiante. Por otro lado, también consideran necesario que el estudiante asuma un papel activo en el programa de aprendizaje-servicio. Por último, destacan la necesidad de reconocer también el trabajo del estudiante en los proyectos de aprendizaje-servicio y que no es recomendable que se vean como actividades complementarias sin reconocimiento.
Acerca de la implicación de la comunidad y las organizaciones con las que se colabora, las tres instituciones destacan querer avanzar en esta dimensión. Más concretamente, reflexionan sobre la importancia de aprender a trabajar en red y hacer sentir a las organizaciones con las que colaboran que son parte de esa red y que son agentes esenciales en el logro de la misión universitaria (conciencia de comunidad). En esta línea, todas las universidades coinciden en querer trabajar para conseguir una mayor sensibilización y conocimiento de las características e implicaciones de la metodología por parte de los socios comunitarios. Además, en las conversaciones con todo el equipo regional se llega a la conclusión de que existen pocas oportunidades para que los socios comunitarios tomen roles de liderazgo en el fomento del aprendizaje-servicio en la universidad.

Respecto a la última dimensión, la que tiene que ver con el apoyo institucional y se recogen los aspectos operativos relacionados con la gestión, organización y administración del proceso, cabe destacar que es necesario seguir trabajando para que haya equipos de trabajos que se encarguen de implementar, desarrollar, e institucionalizar la metodología.

Además, los equipos directivos han de reconocer el aprendizaje-servicio como un objetivo educacional esencial para la universidad y contratar a personas que entienden el aprendizaje-servicio para impulsar la institucionalización. En consecuencia, se llega a la conclusión de que sin recursos económicos disponibles hay pocas oportunidades 
para crear equipos coordinados que impulsen la institucionalización del aprendizaje-servicio solidario.

\section{Conclusiones}

De acuerdo con los resultados obtenidos en este proceso y la evolución positiva que está teniendo el aprendizaje-servicio en nuestro entorno, todavía es necesario seguir avanzando e indagando en el proceso de institucionalización en todas las universidades.

Para ello, tras la imagen global de autoevaluación creada basándonos en las fortalezas y debilidades que hemos ido descubriendo, se ha llevado a cabo un plan de acción por cada universidad.

Por un lado, durante este curso académico participaremos en todas las acciones formativas propuestas desde el proyecto UNISERVITATE, que sirven además de para acompañar la transformación de las instituciones católicas de educación superior a través del aprendizaje-servicio, para tejer redes de colaboración internacionales y formar una comunidad global. Por otro lado, también vamos a llevar a cabo acciones formativas con cada universidad para abordar las necesidades específicas de cada una de ellas. Algunos de los temas a abordar son: cómo crear vínculos estrechos con los socios comunitarios y cómo reconocer institucionalmente al profesorado que participa en experiencias de aprendizaje-servicio. De la misma forma, vemos la necesidad de animar a las tres universidades a enriquecer y dar respuesta a algunas cuestiones que tienen que ver con el proceso de institucionalización a través de las publicaciones que hay en el repositorio UNISERVITATE. Es decir, ser parte de esta práctica global de la que se puede aprender y compartir conocimiento. Por último, queremos proponer a las tres universidades que diseñen un calendario con fechas y acciones que les interese llevar a cabo durante este curso académico.

A modo de conclusión final, nos gustaría destacar que la comunicación entre las distintas universidades va a ser primordial durante este curso para seguir conociendo en qué escenario se sitúa cada una de ellas. Es más, seguiremos dando importancia a crear redes dentro de la región y de manera global continuaremos avanzando en el proceso de institucionalización.

\section{Referencias bibliográficas}

Aktas, C. B., Whelan, R., Stoffer, H., Todd, E., \& Kern, C. L. (2015).

Developing a university- wide course on sustainability: A critical evaluation of planning and implementation. Journal of Cleaner Production, 106(1), 216221.

https://doi.org/10.1016/j.jclepro.2014. 11.037

Asghar, M., \& Rowe, N. (2016). Reciprocity and critical reflection as the key to social justice in service learning: A case study. Innovations in Education and Teaching International, 54(2), 117125.

https://doi.org/10.1080/14703297.201 $\underline{6.1273788}$

Bringle, R.G., \& Hatcher, J. A. (1995). A Service-Learning curriculum for faculty. 
Michigan Journal of Community Service Learning, 2(1), 112-122.

Capella-Peris, C., Gil-Gómez, J., \& Chiva- Bartoll, Ó. (2019). Innovative analysis of Service-Learning effects in physical education: A Mixed-Methods approach. Journal of Research and Practice, 38(7), 638650.https://doi.org/10.1123/jtpe.2019$\underline{003}$

Dewey, J. (1933). How we think: $A$ restatement of the relation of reflective thinking to the education process. Health. https://bit.ly/2Y171vR

Díaz-Iso, A., Eizaguirre, A., \& GarcíaOlalla, A. (2019). Extracurricular activities in higher education and the promotion of reflective learning for sustainability. Sustainability, 11(17), 4521.

https://doi.org/10.3390/su11174521

Freire, P. (2018). Pedagogy of the oppressed. Bloomsbury publishing.

Furco, A. (1996). Service-Learning: A Balanced Approach to Experiential Education. Service-Learning, General, 128. https://bit.ly/3eWViOf

Furco, A. (2003). Rúbrica de autoevaluación para la institucionalización del aprendizajeservicio en la Educación Superior. Revista Internacional sobre investigación en Educación Global y para el desarrollo.

García-Romero, D., y Lalueza, J.L. (2019). Procesos de aprendizaje e identidad en aprendizaje-servicio universitario: Una revisión teórica.
Educación XXI, 22(2), 45-68.

https://doi.org/10.5944/educxx1.22716

Hart, S. (2015). Engaging the learner. The ABC's of service-learning. Teaching and Learning in Nursing, 10(2), 76-79. https://doi.org/10.1016/j.teln.2015.01. $\underline{001}$

Lewing, M., \& Shehane, M. (2017). The institutionalization of service-learning at the independent colleges and universities of the Gulf Coast region. Christian Higher Education, 16(4), 211231.

https://doi.org/10.1080/15363759.201 6.1254074

Mason, M. R., \& Dunens, E. (2019). Service-learning as a practical introduction to undergraduate public health: Benefits for students' outcomes and accreditation. Frontiers in Public Health, 7(63).

https://doi.org/10.3389/fpubh.2019.00 $\underline{063}$

Mezirow, J. (1991). Transformative dimensions of adult learning. JosseyBass.

Páez, J., \& Puig, M. (2013). La reflexión en el Aprendizaje-Servicio. Revista Internacional de Educación para la Justicia, 2 (2), 2254-3139.

https://bit.ly/2Ufo0C6

Pena Mardaras, C. (2020). "A la carta". Diseño de una unidad didáctica para la clase de religión en respuesta a las necesidades de un centro escolar. En Unidad de Innovación Docente, Universidad de Deusto (Ed.), Atendiendo al nuevo perfil del estudiante universitario del siglo XXI 
(pp. 246-252). Grupo de Comunicación Loyola-Bilbao.

Salam, M., Awang Iskandar, D. N., Ibrahim, D. H. A., \& Farooq, M. S. (2019). Service-Learning in higher education: A systematic literature review. Asia Pacific Education Review, 20, 573-593.

https://doi.org/10.1007/s12564-01909580-6

Santos-Pastor, M.L., Cañadas, L., y Martinez-Muñoz, L.F. (2020).

Limitaciones del aprendizaje-servicio en la formación inicial en actividad físicadeportiva. Retos: Nuevas tendencias en educación física, deporte y recreación, 37, 509-517. https://bit.ly/2Y2dj7g

Schön, D.A. (1983). The reflective practitioner: How professionals think in action. Temple Smith.

Song, W., Furco, A., Maruyama, G., \& López, I. (2018). Early exposure to service-learning and college success beyond the freshman year.

International Journal of Research on Service-learning and Community

Engagement, 6(1).

https://bit.ly/3mKq2qw

Sosa, A. (2019). Integración y puesta en práctica de las Preferencias

Apostólicas Universales 2019-2029.

Curia Generalizia della Compagnia di

Gesù.

https://www.jesuits.global/es/uap/

Tapia, M. N. (2010). La propuesta

pedagógica del "Aprendizaje-Servicio": Una perspectiva latinoamericana.

Tzhoecoen, Revista Científica, (5), 2343. https://bit.ly/31QIyFo
UNESCO. (2017). Education for Sustainable development goals: Learning objectives. the Global Education 2030 Agenda. https://bit.ly/32VaPd8

Salterain, I., Eizaguirre, A., Díaz-Iso, A., Aláez Martínez, M., García-Feijoo, M., Roldán-Henao, M., Mota Ribeiro, L., Booth, P. y Simeone, D. (2021). Descripción de un proceso participativo de diagnóstico de la institucionalización del aprendizaje-servicio. RIDAS, Revista Iberoamericana de Aprendizaje Servicio, 12, 149-162. DOI10.1344/RIDAS2021.12.15 